\title{
Some Algorithms Providing Rigourous Bounds for the Eigenvalues of a Matrix
}

\author{
Raymond Pavec \\ LIMI, Université de Bretagne Occidentale \\ Brest,France \\ pavec@univ-brest.fr
}

\begin{abstract}
Three algorithms providing rigourous bounds for the eigenvalues of a real matrix are presented. The first is an implementation of the bisection algorithm for a symmetric tridiagonal matrix using IEEE floating-point arithmetic. The two others use interval arithmetic with directed rounding and are deduced from the Jacobi method for a symmetric matrix and the Jacobi-like method of Eberlein for an unsymmetric matrix.
\end{abstract}

\section{Bisection Algorithm for a Symmetric Tridiagonal Matrix}

Let $A$ be a symmetric tridiagonal matrix of order $n$ :

$$
A=A_{n}=\left(\begin{array}{cccc}
a_{1} & b_{2} & & \\
b_{2} & a_{2} & \ddots & \\
& \ddots & \ddots & b_{n} \\
& & b_{n} & a_{n}
\end{array}\right)
$$

Set $b_{1}=0$, and suppose $b_{k} \neq 0, k=2, \ldots, n$. The bisection method is based on the fact that the sequence $d_{k}(x)$ of principal minors of $A-x I$ is a Sturm sequence:

$$
d_{k}(x)=\operatorname{det}\left(A_{k}-x I_{k}\right), k=1, \ldots, n, \quad d_{0}=1
$$

In floating point arithmetic, as pointed out in [Barth, Martin, Wilkinson 1971], the direct use of this sequence is quite impossible: even for small $n$ underflow and overflow are unavoidable. So they consider (the hypothesis $b_{k} \neq 0, k=2, \ldots, n$ can then be removed):

$$
p_{k}=\frac{d_{k}}{d_{k-1}}, k=1, \ldots, n
$$

This new sequence satisfies the recurrence relations:

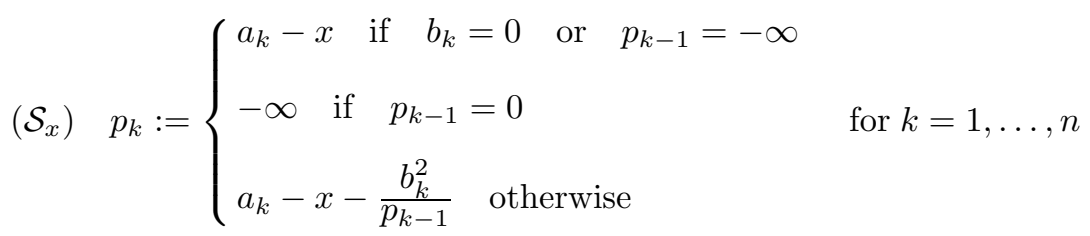


From the Sturm property and the choice of $p_{k}=-\infty$ when $p_{k-1}=0$, it comes that the number of negative terms in $\left(\mathcal{S}_{x}\right)$ is equal to the number of eigenvalues of $A$ smaller than $x$.

Beginning with an interval containing all the eigenvalues of $A$, the method of bisection provides intervals as small as desired, containing the eigenvalues of $A$.

\subsection{Setting it to work in floating point arithmetic}

In floating point arithmetic there are two problems:

- to perform the calculations without overflow,

- to give bounds for the error.

[Barth, Martin, Wilkinson 1971] give bounds for all the eigenvalues. Godunov [Godunov et al. 1993], page 315, gives a similar result but uses a different sequence and guarantees no overflow.

The method presented here (using the sequence $\left(\mathcal{S}_{x}\right)$ ) guarantees the absence of overflow and provides bounds separately for each eigenvalue.

The set of machine-numbers (IEEE standard, available on Macintosh [Apple 1988] or PC compatible [Crawford, Gelsinger 1988]), provides representations for:

$$
\left\{\begin{array}{l}
0,-\infty,+\infty \\
\pm 1, a_{2} \ldots a_{t} 2^{e}, a_{i}=0 \text { or } 1,- \text { min } \leq e \leq \max ,(\text { normalized numbers }) \\
\pm 0, a_{2} \ldots a_{t} 2^{-m i n},(\text { denormalized numbers) }
\end{array}\right.
$$

$t$ is the number of significative digits used. We suppose $\min \leq \max$.

The positive normalized numbers lie in the interval $\left[\epsilon_{0}, M\right]$ :

$$
\epsilon_{0}=2^{-\min }, \quad M=1.1 \ldots 1 \times 2^{\max } \approx 2 \times 2^{\max }
$$

Notice that $\min \leq \max \Rightarrow 1 / \epsilon_{0} \leq 2^{\max } \approx M / 2$.

With rounding to nearest the relative error in usual operations is less than eps $=$ $2^{-t}$, (machine precision). For the machine-operations $\tilde{T}(\top \rightarrow+,-, \times, /)$ we have:

$$
x \tilde{\top} y=(x \top y)(1+\varepsilon),|\varepsilon| \leq e p s
$$

for normalized numbers $x, y$, and $x \top y$. Notice that the presence of denormalized numbers insures that an addition or substraction that gives 0 is exact.

\subsubsection{Calculation without overflow}

In the calculation of $\left(\mathcal{S}_{x}\right)$ the critical term is $b_{k}^{2} / p_{k-1}$. To avoid overflow:

- A scaling of the matrix is performed by multiplying it by $2^{p}, p$ being chosen so that all elements become less than 1 .

- The denormalized numbers occuring during the calculation of $\left(\mathcal{S}_{x}\right)$ are replaced by 0 . This induces an absolute error $\leq \epsilon_{0}$.

Thus, the operations used $(\hat{\top})$ are:

$$
x \hat{\top} y=\left\{\begin{array}{l}
0 \quad \text { if } x \tilde{\top} y \text { is denormalized } \\
x \tilde{\top} y \quad \text { otherwise }
\end{array}\right.
$$


and the calculated sequence is (remember that $b_{1}=0$ ):

$$
\left(\hat{\mathcal{S}}_{x}\right) \quad \hat{p}_{k}:=\left\{\begin{array}{lll}
a_{k} \hat{-} x & \text { if } \quad b_{k}=0 \quad \text { or } \quad \hat{p}_{k-1}=-\infty & \\
-\infty & \text { if } \quad \hat{p}_{k-1}=0 & \text { for } k=1, \ldots, n \\
a_{k} \hat{-} x \hat{-} b_{k} \hat{x} b_{k} \hat{k} \hat{p}_{k-1} & \text { otherwise } &
\end{array}\right.
$$

The eigenvalues of $A$ are therefore in $[-3,3]$, and $x$ also.

If $\hat{p}_{k-1}$ is 0 or infinite there is no problem.

Otherwise $\left|\hat{p}_{k-1}\right|$ is a normalized number $\geq \epsilon_{0}$ :

$$
\left|a_{k}-x-b_{k} \times b_{k} / \hat{p}_{k-1}\right| \leq\left|a_{k}\right|+|x|+1 / \epsilon_{0} \leq 4+2^{\text {min }}<M
$$

and this is also true for machine operations.

\subsubsection{Error estimation}

The scaling of the matrix does not cause any error, except for the elements that become less than $\epsilon_{0}$ in absolute value and are replaced by 0 . The elements $b_{k}^{2}$ that become less than $\epsilon_{0}$ are also replaced by 0 . Thus the matrix becomes $2^{p} A+E^{\prime}$. $E^{\prime}$ is a tridiagonal symmetric matrix which elements are equal to 0 or bounded by $\epsilon_{0}$ or $\sqrt{\epsilon_{0}}$.

$A$ is now the scaled matrix. The classical error analysis ( $\tilde{T}$ operations) shows that the calculation of $\left(\hat{\mathcal{S}}_{x}\right)$ is identical to the calculation of the sequence $\left(\mathcal{S}_{x}\right)$ associated with a matrix $A+E^{\prime \prime}$. This remains true for the $\hat{\top}$ operations. A symmetric tridiagonal matrix $E$ :

$$
E=\left(\begin{array}{cccc}
e_{1} & f_{2} & & \\
f_{2} & e_{2} & \ddots & \\
& \ddots & \ddots & f_{n} \\
& & f_{n} & e_{n}
\end{array}\right)
$$

such that $\left|E^{\prime \prime}\right| \leq E$ will be calculated.

It will be shown that there exist small integers $n a, d a, n b \leq 3$, depending on $k$ such that:

$$
\begin{gathered}
e_{k}=\left|a_{k}-x\right|\left((1+e p s)^{n a}-1\right)+d a \times \epsilon_{0} \\
f_{k}=\left|b_{k}\right|\left((1+e p s)^{\frac{n b}{2}}-1\right)
\end{gathered}
$$

The term $d a \times \epsilon_{0}$ comes from the operations that give 0 or a denormalized result. Let us examine the different cases that occur in the calculation of $\hat{p}_{k}$ and determine the values of the integers $n a, d a$ and $n b$ :

- Case A: $b_{k}=0$ or $\hat{p}_{k-1}=-\infty$.

Then $\hat{p}_{k}=a_{k} \hat{-} x$. Let $y=a_{k} \hat{-} x$. If $y$ is a normalized number we may write:

$$
y=\left(a_{k}-x\right)\left(1+\varepsilon_{1}\right)=a_{k}+\left(a_{k}-x\right) \varepsilon_{1}-x,\left|\varepsilon_{1}\right| \leq \text { eps }
$$

So we have: $\hat{p}_{k}=y, e_{k}=\left|a_{k}-x\right|$ eps, $f_{k}=0 .(n a=1, d a=0, n b=0)$. 
If $y$ is a denormalized number then $\left|a_{k}-x\right| \leq \epsilon_{0}$ and:

$$
\hat{p}_{k}=0=a_{k}-\left(a_{k}-x\right)-x
$$

Thus $e_{k}=\epsilon_{0}, f_{k}=0 .(n a=0, d a=1, n b=0)$.

Lastly if $y=0$, there is no error and $\hat{p}_{k}=0, e_{k}=f_{k}=0 .(n a=d a=n b=0)$.

- Case B: $\hat{p}_{k-1}=0$.

Then $\hat{p}_{k}=-\infty$, and $e_{k}=f_{k}=0 .(n a=d a=n b=0)$.

- Case C: $\hat{p}_{k-1}$ is a normalized number.

For each operation three cases (at the most) are to be considered according to the result: 0 , denormalized (replaced by 0 ), normalized.

- Usual case C0: In the usual case all operations give a normalized result and we get:

$$
\hat{p}_{k}=\left(\left(a_{k}-x\right)\left(1+\varepsilon_{1}\right)-\frac{b_{k}^{2}\left(1+\varepsilon_{2}\right)}{\hat{p}_{k-1}}\left(1+\varepsilon_{3}\right)\right)\left(1+\varepsilon_{4}\right),\left|\varepsilon_{i}\right| \leq e p s
$$

The $\varepsilon_{i}$ are the relative errors occurring in the operations. Thus:

$$
\hat{p}_{k}=a_{k}+\left(a_{k}-x\right)\left(\varepsilon_{1}+\varepsilon_{4}+\varepsilon_{1} \varepsilon_{4}\right)-x-\frac{b_{k}^{2}\left(1+\varepsilon_{2}\right)\left(1+\varepsilon_{3}\right)\left(1+\varepsilon_{4}\right)}{\hat{p}_{k-1}}
$$

This is the exact result corresponding to:

$$
a_{k}+\left(a_{k}-x\right)\left(\varepsilon_{1}+\varepsilon_{4}+\varepsilon_{1} \varepsilon_{4}\right) \text { and } b_{k}^{2}\left(1+\varepsilon_{2}\right)\left(1+\varepsilon_{3}\right)\left(1+\varepsilon_{4}\right)
$$

Thus in this case:

$$
\begin{gathered}
e_{k}=\left|a_{k}-x\right|\left(2 e p s+e p s^{2}\right),(n a=2, d a=0) \\
f_{k}=\left|b_{k}\right|\left(\sqrt{(1+e p s)^{3}}-1\right),(n b=3)
\end{gathered}
$$

- Case C1: $b_{k} \tilde{\times} b_{k} \tilde{/} \hat{p}_{k-1}$ gives 0 or a denormalized result (and thus replaced by 0$)$. In this case:

$$
\hat{p}_{k}=a_{k} \hat{-} x
$$

So we may write:

$$
\hat{p}_{k}=a_{k} \hat{-x}+\left[\frac{b_{k} \tilde{x} b_{k}}{\hat{p}_{k-1}}\right]-\frac{b_{k}^{2}\left(1+\varepsilon_{2}\right)}{\hat{p}_{k-1}}
$$

and in this case we have to add $\epsilon_{0}$ to the $e_{k}$ obtained in case $\mathbf{A}$ (add 1 to $d a$ ) and $n b=1$.

- Case C2: $a_{k}-x=0$. Excluding case $C 1$ we have:

$$
\hat{p}_{k}=-\frac{b_{k}^{2}\left(1+\varepsilon_{2}\right)\left(1+\varepsilon_{3}\right)}{\hat{p}_{k-1}}
$$

and thus: $e_{k}=0,(n a=0, d a=0), \quad f_{k}=\left|b_{k}\right| e p s,(n b=2)$. 
- Case C3: $a_{k} \tilde{-} x$ is denormalized. This case is the same as C2, except that $e_{k}=\epsilon_{0},(n a=0, d a=1, n b=2)$.

$\circ$ Case $C_{4}$ : All operations give a normalized result, except the last substraction. If the result is 0 , then consider the usual case and set $\varepsilon_{4}$ to 0 . It comes:

$$
e_{k}=\left|a_{k}-x\right| e p s, f_{k}=\left|b_{k}\right| e p s,(n a=1, d a=0, n b=2)
$$

If the result is denormalized add $\epsilon_{0}$ to $e_{k},(n a=1, d a=1, n b=2)$.

\subsection{Bounds for the eigenvalues}

Naming $\lambda_{i}(X), i=1, \ldots, n$, the eigenvalues of a symmetric matrix $X$ of order $n$, in increasing order, we have [Wilkinson 1965], page 101:

$$
\lambda_{i}(A)+\lambda_{1}\left(E^{\prime \prime}\right) \leq \lambda_{i}\left(A+E^{\prime \prime}\right) \leq \lambda_{i}(A)+\lambda_{n}\left(E^{\prime \prime}\right)
$$

Let $\rho$ be the spectral radius of $E^{\prime \prime}$. We get:

$$
\lambda_{i}(A)-\rho \leq \lambda_{i}\left(A+E^{\prime \prime}\right) \leq \lambda_{i}(A)+\rho
$$

and also:

$$
\lambda_{i}\left(A+E^{\prime \prime}\right)-\rho \leq \lambda_{i}(A) \leq \lambda_{i}\left(A+E^{\prime \prime}\right)+\rho
$$

$\rho$ is bounded by $\left\|E^{\prime \prime}\right\|_{\infty}$, and by $\|E\|_{\infty}$.

Let $m_{x}$ be the number of negative terms in $\left(\hat{\mathcal{S}}_{x}\right)$. We have:

$$
\begin{gathered}
\lambda_{i}\left(A+E^{\prime \prime}\right)<x, i=1, \ldots, m_{x} \\
\lambda_{i}\left(A+E^{\prime \prime}\right) \geq x, i=m_{x}+1, \ldots, n
\end{gathered}
$$

Naming $\delta_{x}$ the infinite norm of $E$, it comes:

$$
\begin{gathered}
\lambda_{i}(A)<x+\delta_{x}, i=1, \ldots, m_{x} \\
\lambda_{i}(A) \geq x-\delta_{x}, i=m_{x}+1, \ldots, n
\end{gathered}
$$

If the interval $[x, y]$ is such that $k=m_{y}-m_{x}>0$, we may say that:

$$
\lambda_{i}(A) \in\left[x-\delta_{x}, y+\delta_{y}\left[, i=m_{x}+1, \ldots, m_{y}\right.\right.
$$

It remains to take into account the scaling of the matrix and the initial rounding errors.

The bisection algorithm gives intervals such that $m_{x}<m_{y}$ : to determine $\lambda_{k}(A)$ for a given $k$ first choose $[x, y]$ such that $m_{x}<k \leq m_{y}$ and calculate $z=(x \hat{+} y) / 2$ and $m_{z}$ :

- if $m_{z} \geq k$ the new interval will be $[x, z]$,

- if $m_{z}<k$ the new interval will be $[z, y]$. 


\subsection{Algorithm and example}

The general structure of the algorithm is the same as in [Barth, Martin, Wilkinson 1971], except the final test: for each eigenvalue the bisection process is continued until the interval $[x, y]$ is such that $x$ and $y$ are two consecutive machinenumbers (denormalized numbers excluded), or until a fixed maximum number of steps is reached.

The machine precision used is eps $=2^{-64} \approx 5,5 \times 10^{-20}$. This precision is available on micro-computers [Apple 1988], [Crawford, Gelsinger 1988]. The results have been obtained using the software [Pavec 1994].

Consider the matrix of order $n=30$ from [Barth, Martin, Wilkinson 1971]:

$$
a_{i}=i^{4}, b_{i}=i-1, \quad i=1, \ldots, 30
$$

The eigenvalues lie in the interval $\left[0.933 . ., 8.100 . . \times 10^{5}\right]$. A few results are shown in the table below:

- the bounds for the absolute error ' $\Delta \lambda_{i}$ ' and relative error ' $\Delta \lambda_{i} / \lambda_{i}$ ' take in account the final rounding from base 2 to base 10 ,

- 'Steps' is the number of bisection steps performed and is less than the fixed maximum $(=100)$.

\begin{tabular}{|c|c|c|c|c|}
\hline$i$ & $\lambda_{i}$ & $\Delta \lambda_{i}$ & $\Delta \lambda_{i} / \lambda_{i}$ & Steps \\
\hline 30 & $8.100000081873846690 \times 10^{5}$ & $2.3 \times 10^{-13}$ & $2.9 \times 10^{-19}$ & 64 \\
\hline 20 & $1.600000005628909621 \times 10^{5}$ & $1.4 \times 10^{-13}$ & $8.8 \times 10^{-19}$ & 62 \\
\hline 10 & $1.000000200627702490 \times 10^{4}$ & $9.3 \times 10^{-14}$ & $9.3 \times 10^{-18}$ & 64 \\
\hline 1 & 0.933407084865963 & $8.8 \times 10^{-14}$ & $9.5 \times 10^{-14}$ & 68 \\
\hline
\end{tabular}

\section{Symmetric Matrices and Jacobi's Algorithm}

Jacobi's method [Rutishauser 1991] applied to a symmetric matrix $A$ constructs a sequence of symmetric matrices $A_{k}$, similar to $A$, and converging to a diagonal matrix:

$$
A_{k+1}=R_{k}^{T} A_{k} R_{k}
$$

where $R_{k}$ is a rotation (angle $\theta_{k}$ ) matrix calculated to annihilate a non diagonal element $(i, j)$ of $A_{k} \cdot t_{k}=\tan \theta_{k}$ is obtained from $a_{i i}^{(k)}, a_{i j}^{(k)}, a_{j j}^{(k)}$.

\subsection{Setting it to work on matrices of intervals}

In order to obtain rigourous bounds for the eigenvalues, we work on matrices of intervals.

The elementary operations $(+,-, \times, /, \sqrt{ })$ on intervals [Moore 1979] are performed using directed rounding provided by the IEEE standard, without using the extensions developped in [Kulisch, Miranker 1981].

$A$ number or a matrix $x$ represented by an interval is written $[x]$. 
Let $[A]$ be the initial symmetric matrix of intervals. Starting with $[A]_{1}=[A]$, we generate a sequence of symmetric matrices $[A]_{k}$ such that all symmetric matrix $B$ belonging to $[A]$ is similar to a symmetric matrix $B_{k}$ belonging to $[A]_{k}$.

Thus the eigenvalues of $B$ are the eigenvalues of a symmetric matrix $B_{k}$ belonging to $[A]_{k}$.

For an interval matrix $[A]_{k}$, in order to restrict the growth of the intervals, a machine-number $t_{k}$ is calculated on the midpoint of the intervals $[a]_{i i}^{(k)},[a]_{i j}^{(k)}$ and $[a]_{j j}^{(k)}$, using ordinary floating point arithmetic.

The corresponding rotation is:

$$
R_{k}=\left(\begin{array}{rr}
c & -s \\
s & c
\end{array}\right) \quad c=1 / \sqrt{1+t_{k}^{2}}, s=t_{k} c
$$

Using interval arithmetic to evaluate $c$ and $s$, we get an interval matrix $[R]_{k}$ containing $R_{k}$ and we set:

$$
[A]_{k+1}=[R]_{k}^{T}[A]_{k}[R]_{k}
$$

If $B$ is a symmetric matrix belonging to $[A]$ then

$$
B_{k}=R_{k-1}^{T} \ldots R_{1}^{T} B R_{1} \ldots R_{k-1}
$$

is similar to $B$ and belongs to $[A]_{k}$.

Notice that the sequence $[A]_{k}$ does not necessarily contain the sequence generated by Jacobi's method, and that the element $[a]_{i j}^{(k)}$ is not annihilated but replaced by a small interval.

These rotations are performed in a cyclic way, among the elements that do not contain 0 .

\subsection{Bounds for the eigenvalues}

Let $D_{k}$ be the diagonal matrix which diagonal elements are the midpoint of the elements of $[A]_{k}$, and $[C]_{k}$ a matrix such that:

$$
[A]_{k} \subseteq D_{k}+[C]_{k}
$$

The theorem of Gershgorin [Wilkinson 1965] shows that the eigenvalues of a symmetric matrix belonging to $[A]$ are in the union of the closed intervals:

$$
\left[d_{i}-r_{i}, d_{i}+r_{i}\right], \quad r_{i}=\sum_{j=1}^{n}\left|[c]_{i j}\right|
$$

where $\left|[c]_{i j}\right|$ is:

$$
\left|[c]_{i j}\right|=\max _{t \in[c]_{i j}}|t|
$$




\section{$2.3 \quad$ Examples}

- Condider the matrix [Wilkinson, Reinsch, 1991], page 223:

$$
A=\left(\begin{array}{rrrrr}
10 & 1 & 2 & 3 & 4 \\
1 & 9 & -1 & 2 & -3 \\
2 & -1 & 7 & 3 & -5 \\
3 & 2 & 3 & 12 & -1 \\
4 & -3 & -5 & -1 & 15
\end{array}\right)
$$

This matrix is stored as zero-length intervals. After a sweep of the lower triangle the Gershgorin's disks are (radius $\leq 2.1$ ):

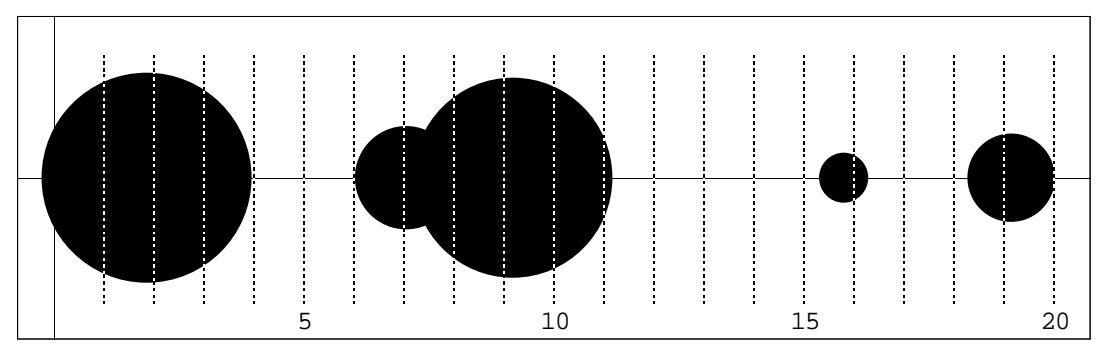

Figure 1: Gershgorin disks after a sweep

Going on with the sweeps until all non-diagonal elements contain 0 , the radii of all the disks (Machine precision: $2^{-64}$ ) become successively less than:

$$
0.073, \quad 1.67 \times 10^{-5}, \quad 2.25 \times 10^{-16}
$$

- The matrix $B=8 J-5 J^{2}+J^{3}$ of order 44 [Rutishauser 1991], page 209, where $J$ denotes the tridiagonal matrix with $J_{i i}=2, J_{i, i+1}=1$, has eleven eigenvalues in the interval $[4,4.163]$, the length of which is $1 / 100$ of the total length of the spectrum.

After 7 sweeps all the eigenvalues are separated, except two that lie in the interval $[4.0032,4.0065]$.

\section{Eberlein's Jacobi-like Method for Unsymmetric Real Matrices}

For an unsymmetric real matrix $A$ Eberlein gives a Jacobi-like method [Eberlein 1962]:

$$
A_{k+1}=T_{k}^{-1} A_{k} T_{k}
$$

$A_{k}$ generally converges to a block-diagonal matrix, with blocks of order 1 or 2 . The blocks of order two have complex conjugate eigenvalues $a \pm i b$ :

$$
\left(\begin{array}{rr}
a & -b \\
b & a
\end{array}\right)
$$

$T_{k}$ is the product of a rotation $R_{k}$ and of a matrix $S_{k}$ : 


$$
S_{k}=\left(\begin{array}{rr}
\cosh y & -\sinh y \\
-\sinh y & \cosh y
\end{array}\right)
$$

working in a coordinate plane $(i, j)$.

\subsection{Bounds for the eigenvalues}

After some sweeps of Eberlein's method we expect a matrix of the form:

$$
\Delta+E
$$

where $\Delta$ is a block-diagonal matrix, and $E$ a matrix with small elements. The following theorem, that is a particular case of the theorem of Bauer-Fike [Golub, Van Loan 1983], page 342, gives disks of the complex plane containing the eigenvalues of $\Delta+E$ :

Theorem. Let $A=\Delta+E$, where $\Delta$ is a block-diagonal with blocks of order 1 or 2.

$$
\begin{aligned}
& \Delta=\operatorname{diag}\left(\Delta_{1}, \Delta_{2}, \ldots, \Delta_{p}\right) \\
& \Delta_{r}=\alpha_{r} \quad \text { or } \quad\left(\begin{array}{rr}
\alpha_{r} & -\beta_{r} \\
\beta_{r} & \alpha_{r}
\end{array}\right)
\end{aligned}
$$

$\Delta$ can be diagonalised with a unitary block-diagonal matrix $X$ which has the same structure as $\Delta$. Let $F=X^{-1} E X$. Then the eigenvalues of $A$ are in the disks of the complex plane, which centers are the eigenvalues of $\Delta$ and the radii:

$$
r_{i}=\sum_{j=1}^{n}\left|f_{i j}\right|
$$

If the union $U$ of $k$ of these disks is isolated from the others then $U$ contains exactly $k$ eigenvalues of $A$.

Proof. The blocks of order 1 of $X$ are equal to 1 and the blocks of order 2 to:

$$
X_{r}=\frac{1}{\sqrt{2}}\left(\begin{array}{rr}
i & -i \\
1 & 1
\end{array}\right), \quad X_{r}^{-1}=\frac{1}{\sqrt{2}}\left(\begin{array}{rr}
-i & 1 \\
i & 1
\end{array}\right)
$$

Let $\lambda_{1}, \ldots, \lambda_{n}$ be the eigenvalues of $\Delta$ and

$$
\Delta^{\prime}=X^{-1} \Delta X=\operatorname{diag}\left(\lambda_{1}, \ldots, \lambda_{n}\right)
$$

If $\lambda$ is an eigenvalue of $A$, then $A-\lambda I$ is singular, and

$$
X^{-1}(A-\lambda I) X=\Delta^{\prime}-\lambda I+F
$$

also. There exists a complex vector $x,\|x\|_{\infty}=1$, such that:

$$
\left(\Delta^{\prime}-\lambda I\right) x=-F x
$$


Thus

$$
\left(\lambda_{i}-\lambda\right) x_{i}=-\sum_{j=1}^{n} f_{i j} x_{j}, i=1, \ldots, n
$$

Choosing $i$ such that $\left|x_{i}\right|=1$ we get:

$$
\left|\lambda_{i}-\lambda\right| \leq \sum_{j=1}^{n}\left|f_{i j}\right|=r_{i}
$$

$r_{i}$ is easily expressed in terms of $E$.

If the union $U$ of $k$ of these disks is isolated from the union $V$ of the others, the disks associated with $A(t)=\Delta+t E$ have the same property for all $t \in[0,1]$ : the centers do not change and the radii are $t r_{i}$.

The result comes then from the fact that $U$ contains exactly $k$ eigenvalues of $A(0)$, and $V$ exactly $n-k$. The eigenvalues of $A(t)$ are continuous functions of $t$, so they cannot jump from $U$ to $V$.

\subsection{Setting it to work on matrices of intervals}

A sequence of matrices $[A]_{k}$ is generated from the initial matrix $[A]$, such that each matrix $B \in[A]$ is similar to $B_{k} \in[A]_{k}$.

At each stage of the method $T_{k}=R S$ is calculated using ordinary floating point arithmetic and the midpoints of the elements of $[A]_{k}$.

Then

$$
\left[T_{k}^{-1}\right] \text { and }[A]_{k+1}=\left[T_{k}^{-1}\right][A]_{k} T_{k}
$$

are calculated using interval arithmetic.

It remains then to decompose $[A]_{k}$ under the form:

$$
[A]_{k} \subseteq \Delta_{k}+[C]_{k}
$$

where $\Delta_{k}$ is a block-diagonal matrix and to calculate disks containing the eigenvalues. Bounds for the radii of these disks are deduced from the theorem of the preceding section.

\subsection{Examples}

- Consider the matrix $A$ [Eberlein, Boothroyd 1971], page 334:

$$
A=\left(\begin{array}{rrrr}
6 & -3 & 4 & 1 \\
4 & 2 & 4 & 0 \\
4 & -2 & 3 & 1 \\
4 & 2 & 3 & 1
\end{array}\right)
$$

$A$ has two double eigenvalues equal to $3 \pm \sqrt{5}$ and is defective. 
The three first sweeps give:
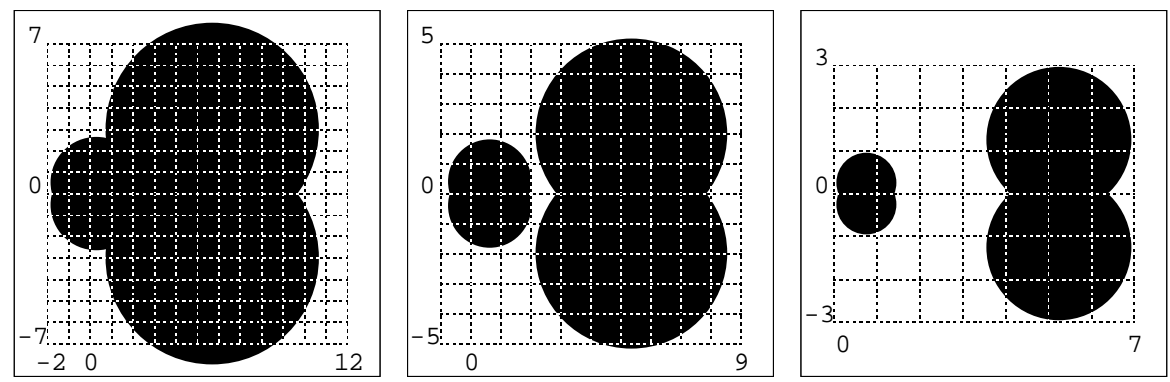

Figure 2: The three first sweeps

After 2 sweeps the disks are included within to disjoint regions.

Finally we get disks whith radii bounded by $3,98 \times 10^{-8}$ and $2,2 \times 10^{-8}$.

- For the matrix $B=\left(b_{i j}\right)$ of order 12 [Eberlein, Boothroyd 1971], page 334, defined by:

$$
\left\{\begin{array}{l}
b_{i j}=13-j, \text { if } i \leq j \\
b_{i, j}=12-j, \text { if } i=j+1 \\
b_{i j}=0, \text { if } i>j+1
\end{array}\right.
$$

after 33 sweeps the 12 disks are disjoint and the radii are $<0.001$.

\section{Conclusion}

These algorithms are implemented in an interactive software [Pavec 1994] working on the Apple Macintosh series of micro-computers. This software is devoted to:

- teaching of mathematics [Pavec 1993]: a very simple Pascal style language lets you create interactively changeable figures containing curves, text, numerical values ...

- and teaching of elementary numerical analysis: interpolation, approximation, ordinary differential equations, numerical integration, systems of equations, eigenvalues.

A commented Pascal version of the algorithm of bisection is available by email(pavec@univ-brest.fr).

\section{References}

[Apple 1988] "Apple Numerics manual"; Addison-Wesley (1988).

[Barth, Martin, Wilkinson, 1971] Barth, W., Martin, R.S., Wilkinson, J.H.: "Calculation of the eigenvalues of a symmetric tridiagonal matrix by the method of bisection"; in [Wilkinson, Reinsch, 1991], 249-256.

[Crawford, Gelsinger 1988] Crawford, J.H., Gelsinger, P.P.: "Programmation du 80386"; Sybex (1988).

[Eberlein 1962] Eberlein, P.J.: "A Jacobi-like method for the automatic computation of eigenvalues and eigenvectors of an arbitrary matrix"; J. Soc. Indus. Appl. Math., 10, 74-88 (1962). 
[Eberlein, Boothroyd 1971] Eberlein, P.J., Boothroyd, J.: "Solution to the eigenproblem by a norm reducing Jacobi type method"; in [Wilkinson, Reinsch, 1971], 327-338.

[Godunov et al., 1993] Godunov, S.K., Antonov, A.G., Kiriljuk, O.P., Kostin, V.I.: "Guaranteed accuracy in numerical linear algebra"; Kluwer Academic Publishers (1993).

[Golub, Van Loan 1983] Golub, G.H., Van Loan, C.F.: "Matrix Computations"; The Johns Hopkins University Press (1983).

[Kulisch, Miranker 1981] Kulisch, U.W., Miranker, W.L.: "Computer Arithmetic in Theory and Practice"; Academic Press (1981).

[Moore 1979] Moore, R.E.: "Methods and applications of interval analysis"; S.I.A.M. (1979).

[Pavec 1993] Pavec, R.: "Maths\&Calculs-io, a software for teaching, combining graphic representations and numerical methods"; Proc. French-Norwegian Symposium on Computer-Aided Mathematics, (1993).

[Pavec 1994] Pavec, R.: "Logiciel Maths\&Calculs-io"; Editions JoliCiel, Noisiel, France (1994).

[Rutishauser 1971] Rutishauser, H.: "The Jacobi method for real symmetric matrices" in [Wilkinson, Reinsch, 1991], 201-211.

[Wilkinson 1965] Wilkinson, J.H.: "The algebraic eigenvalue problem"; Clarendon Press. Oxford (1965)

[Wilkinson, Reinsch, 1971] Wilkinson, J.H., Reinsch, C.: "Handbook for Automatic Computation, Volume II: Linear Algebra"; Springer Verlag. Berlin, Heidelberg, New York (1971). 